\title{
Shifting habitats
}

\author{
Climate change is altering environmental niches, causing species to shift their habitat range as they track their \\ ecological niche. These shifts allow species to persist but may disadvantage existing species in these areas; \\ understanding the positives and negatives is needed to ensure effective management for biodiversity.
}

S pecies (both plants and animals) are being affected by climate change, and as they reach their ecological limit, they need to shift their habitat to survive. Climate is not the only driver of habitat shifts: other human activities, such as urban or infrastructure development, and more broadly changing land uses may also drive shifts in habitat. However, climate change will accelerate range shifts, and identifying species habitats and whether change is occurring is difficult, as there may be a lack of data to define the historical range of a species or it may have been non-static during the recent past.

Range shift for plant species may be more detectable, as plant species tend to shift more slowly; however, plant species may change latitude or elevation in response to climate changes (Science 292, 673-679; 2001). For all species, as warming continues, poleward expansion and equatorward contraction of ranges is to be expected, with marine species having the potential to retain the equatorial end of their range as they can shift deeper to escape warmer surface waters.

Individual species' tolerance to environmental change will determine their need to shift, and to which extent. For example, a European-wide study of bird and butterfly communities spanning from 1990 to 2008 (Nat. Clim. Change 2, 121-124; 2012) showed that butterflies had moved further northward in that time than bird species - a community shift equivalent to $114 \mathrm{~km}$ compared to $37 \mathrm{~km}$ for bird communities. Incoming species can also cause community composition and structure to be altered, which may result in further relocations or pressures on inhabitants.

Presently, there has been a strong focus on the positives of range shifts ensuring species' survival; however, there are also negative impacts of climate-driven range shifts, which Piper Wallingford, Toni Lyn Morelli and colleagues address in a Review in this issue. They suggest that an invasive species risk assessment framework can be used to help acknowledge these. Adapting such a framework allows similarities to be found between invasive species and climate-driven range shifters in order to facilitate management and survival of both

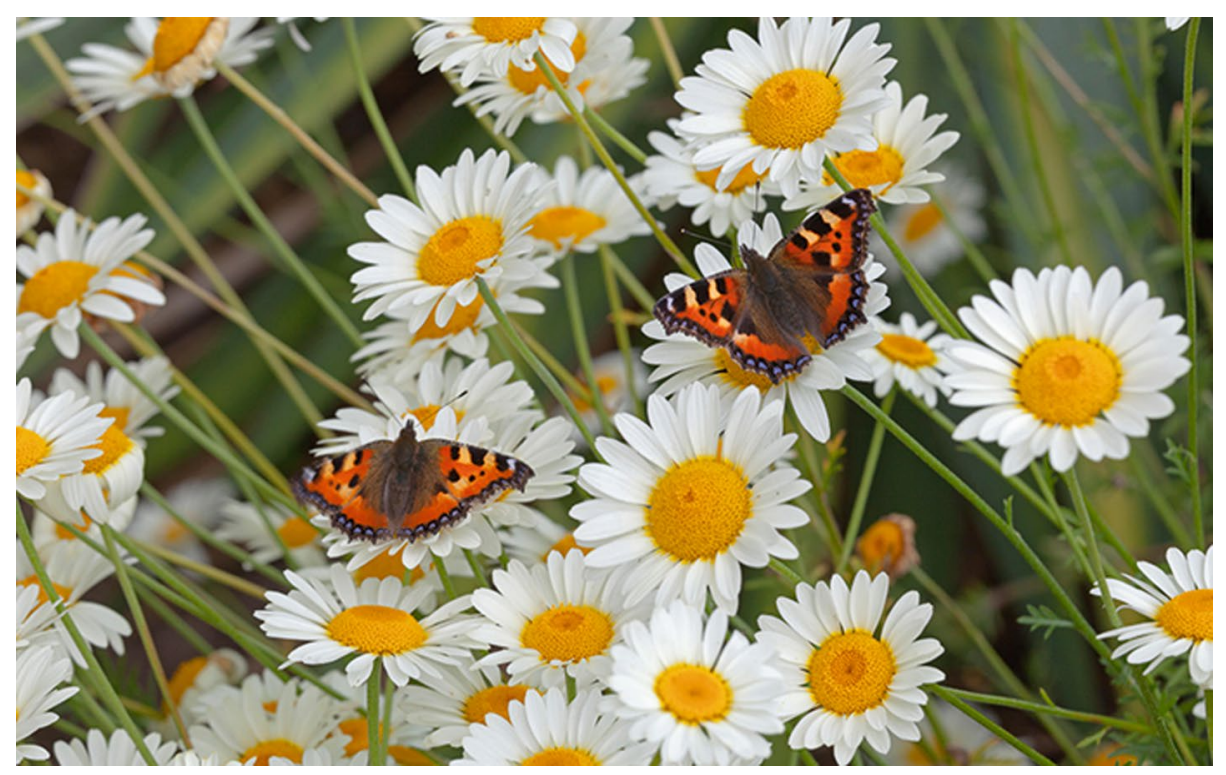

Credit: Ernie Janes / Alamy Stock Photo

the species shifting into an area and the original inhabitants.

A counter view is also presented in this issue: Mark Urban writes in a Comment that while invasive species and climate change-driven range-shifting species result from human disturbance, there are differences which reduce the usefulness of such a comparison. Urban notes that trait-based approaches to predict which species will shift in response to climate change have not been useful, and that there is a need for more observation as well as experimental work to understand species' climate change responses.

A combination of these views are reflected in a Nature Climate Change Perspective from 2019, where Brett Scheffers and Gretta Pecl encourage more collaborative monitoring and management of species as climate-driven shifts result in relocations across geopolitical boundaries (Nat. Clim. Change 9, 581-586; 2019). They identify that shifting species fall into three categories: persecuted, protected, and overlooked or ignored. The persecuted are those that are considered invaders and may impact on existing ecosystem services or agriculture, potentially incurring costs to control or eradicate. The protected, on the other hand, would have protected status that is recognized in the new habitat; however, knowing a species has established in a new location is needed for this information to be publicly distributed. This is similar to the overlooked, where small numbers are identified but may be considered outside of their known habitat, rather than that habitat having already shifted or being in the process of shifting.

How to understand climate change effects of species and their interactions as they seek out their individual niche is a complex question. Will lessons from the past help, or is the current change too rapid? The Galapagos Islands, Darwin's natural laboratory, as discussed in a Comment, could still help understand ecosystem responses and adaptation today. There will be winners and losers as species seek out their niche, but management needs to be adaptive and dynamic to the constant change to provide the best protections for biodiversity as a whole.

Published online: 30 April 2020 https://doi.org/10.1038/s41558-020-0789-x 